Copyright (C) 2015 IEEE. Personal use of this material is permitted. Permission from IEEE must be obtained for all other uses, in any current or future media, including reprinting/republishing this material for advertising or promotional purposes, creating new collective works, for resale or redistribution to servers or lists, or reuse of any copyrighted component of this work in other works. 


\title{
EMC Applications for Military: Reverberation Chamber Tests
}

\author{
Chittawan Choeysakul***, Pakorn Rattanakreep***, Franz Schlagenhaufer**, Peter Hall** \\ **ICRAR/Curtin Institute of Radio Astronomy \\ Curtin University, Western Australia, Australia \\ *** Royal Thai Navy \\ Bangkok, Thailand
}

*Chittawan.choeysakul@postgrad.curtin.edu.au

\begin{abstract}
Electrical and electronic equipment installed on military platforms must have very low electromagnetic emission and good immunity for the whole operational frequency range. Reverberation Chambers (RC) are tools for sensitive emission measurements and immunity tests against strong electromagnetic fields, at a lower cost than other techniques. Method of RC should be suitable for testing Military's electronic devices such as radio or radar system. However, RCs must be large for tests at low frequencies; for example, at $80 \mathrm{MHz}$ are conventional $\mathrm{RC}$ must have dimensions up to $7 \mathrm{~m}$ by $15 \mathrm{~m}$ by $8 \mathrm{~m}$. For military concern, the lowest operation frequency can be as low as $2 \mathrm{MHz}$ (underwater communication can be lower).

Conventional RCs can only be used above a certain frequency, the lowest usable frequency (LUF), as they require a minimum mode density (number of modes per frequency interval) in order for the stirrer to perform effectively and alter field distributions. Technique of MIMO RC $[1,2]$ can make RCs usable down to much lower frequencies; it can mean the dimensions of the chamber can be up to 6 times smaller.

However, the composite Q-factor of RCs can be rather low at low frequencies, and this affects the sensitivity, and ultimately usability of an RC. This paper studies the possibility to increase composite $Q$-factor when $\mathrm{RC}$ is used at lower frequencies than conventional method.
\end{abstract}

Keywords-Reverberation chambers (RC), emission measurements, $Q$-factor, $L U F$

\section{INTRODUCTION}

By 1967, Electromagnetic Compatibility (EMC) had been recognised as an issue in the integration of electronic systems, which had little connection between standards developers in different parts of the world, most nations had no product-level regulation and different services used different specifications. In 1967, the_USS Forrestal disaster has brought EMC to the front of military minds. The USS Forrestal was an aircraft carrier operating in the Vietnam War, where it used F-4 Phantom aircraft loaded with Zuni rocket, which used an electrical signal to activate launch. Accidentally, in July 1967, one of the F-4 Phantoms on the carrier's deck inadvertently launched a Zuni rocket, which crossed the deck and struck the full belly fuel tank of another aircraft. The ensuing explosion and fire of the aircraft's $1000 \mathrm{lb}$ bomb caused a chain reaction of fires and explosions resulting in ultimately the loss of 134 sailors and the injury of a further 62 [3]. The flight deck was penetrated by the heat and explosions, causing fire to spread throughout the ship; the fires continued to burn on three levels below the flight deck for many hours afterwards.

Although the actual cause is unknown, two possibly assumptions of the incident are related to EMC. Firstly, it was a problem of shielding effectiveness, as a faulty cable shield allowed the extremely high power ship's transmitters to the launch command lines [4]. Second thought was an electrical transient happened during weapon loading resulting in activating of a false launch signal. EMC is a key suspect for both assumptions.

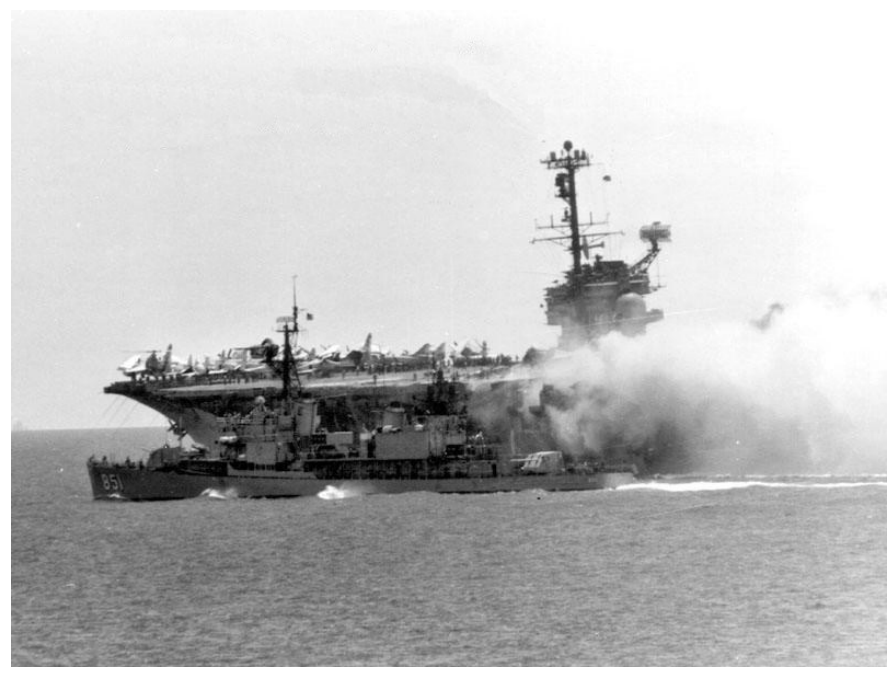

Fig. 1: The USS Forrestal disaster in 1967 related to EMC [3].

After that, military standards on EMC were released in USA At the centre were MIL-STD 461, 462 and 463.MILSTD 461 is still in use, and the recent update is MIL-STD$461 \mathrm{~F}$ from 2007 [5]; Militaries around the world also use MIL-STD-461F, unless they have developed their own standards. MIL-STD-461F provides both recommended test levels and the test procedures for a number of different tests. These are divided into four broad categories: CE - Conducted Emissions, CS - Conducted Susceptibility, RE - Radiated Emissions, and RS - Radiated Susceptibility. The requirements are drawn from the standard and shown as Fig. 2. Obviously, from the tables, the requirements for Navy Surface ships and submarines are the most demanding compare to Army and Air Force applications.

\section{REVERBERATION CHAMBER FOR THE REQUIREMENTS}

MIL-STD461f [5] is also used as reference for Royal Thai Military and many countries. To demonstrate compliance emission measurements and susceptibility tests must be performed; there are four main methods for radiated emission and susceptibility tests (RE and RS). Method of Reverberation Chamber (RC) is a test in an electrically-large, highly conductive, resonant cavity [6]. Emission measurements can 
be made more sensitive compared with an OATS or AC, and immunity tests can also be done at low cost because high field strengths can be generated with a moderate amplifier power. RC method provides a statistical result which is good for stability, repeatability and reliability. Besides, cost per square meters of $\mathrm{RC}$ is cheaper than $\mathrm{AC}$ (no need for any absorber).

For making emission measurements, $\mathrm{RC}$ method is only one method that has capable to measure indirect path loss by controlling the reflecting signals, while the others are measuring direct path loss by controlling the environment. As the result, normal method (for instance OATS) has some common limitations (figure 4) related to high ambient noise level, reflections, weather, and emission masking; RC can find problems, as emission problems cannot be hidden.

A high quality factor (Q) allows generating strong signals with very low source power, or measuring the emission from weak emission with conventional EMC receivers. The benefit also applies to immunity tests. This is important for military equipment on deck/platform which can be subject to high field strength at low frequencies (HF transmitters) up to very high frequencies (RADAR system). Besides, from my point of view, method of RC could be suitable for simulating a hybrid environment as most rooms on the ship or tank will probably have similar characteristics as resonant cavities.

Conceptually, method of RC is stirring standing waves, as they occur in an enclosure, by using a metallic paddle (called stirrer) to change boundary conditions for, and such change the spatial distribution of electromagnetic fields inside the cavity. However, the lowest usable frequency (LUF) is one of the most concerns for every $\mathrm{RC}$, as the chamber can only be used above a certain frequency. The LUF is affected by composite Q-factor; higher composite Q-factor can affect in having more sensitive emission measurements but also resulting in higher the LUF.

According to [6], the LUF for a conventional RC (i.e. one which is used according to IEC 61000-4-21 [6] can be predicted by (1), or (2) with approximation of the $60^{\text {th }}$ mode with a rectangular empty cavity with dimensions $\mathrm{L}, \mathrm{W}$, and $\mathrm{H}$ (in $\mathrm{m}$ ), with no information of composite Q-factor. The LUF (in Hz) can be calculated by:

$$
\begin{aligned}
& N \approx \frac{8 \pi}{3} L W H \frac{f^{3}}{c^{3}}-(L+W+H) \frac{f}{c}+\frac{1}{2} \\
& F_{l, m, n}=\frac{c}{2} \sqrt{\left[\left(\frac{l}{L}\right)^{2}+\left(\frac{m}{W}\right)^{2}+\left(\frac{n}{H}\right)^{2}\right]} ; \quad \mu_{r}, \epsilon_{r}=1 ;
\end{aligned}
$$

where $c$ is the speed of wave propagation in the enclosure $(\mathrm{m} / \mathrm{s}) . l, m$, and $n$ are the mode indices (at least two of them are nonzero). $N$ is number of mode.

Operational frequencies for the Royal Thai Navy cover a very wide range; HTMS Makutrajakumarn as an example, uses frequencies from $\mathrm{HF}(2 \mathrm{MHz}$ ) to $\mathrm{Ku}$ band (up to $18 \mathrm{GHz}$ ). To be usable at frequencies down to just $30 \mathrm{MHz}$ would require a conventional $\mathrm{RC}$ with dimensions in the order of 30 $\mathrm{m}$ by $11 \mathrm{~m}$ by $6 \mathrm{~m}$ [7]. Lower frequencies would require even larger chambers. A special technique developed at the International Centre for Radio Astronomy Research (ICRAR) at Curtin University, Western Australia, [1,2] allows RC to be used at much lower frequencies [8]; the technique is called Multiple Input Multiple Output RC (MIMO RC) (see Fig. 6). As the MIMO RC is used at much lower frequencies than a conventional $\mathrm{RC}$, the quality factor of a resonant cavity at low frequencies must be considered, and this is this paper's topic.

TABLE V. Requirement matrix.

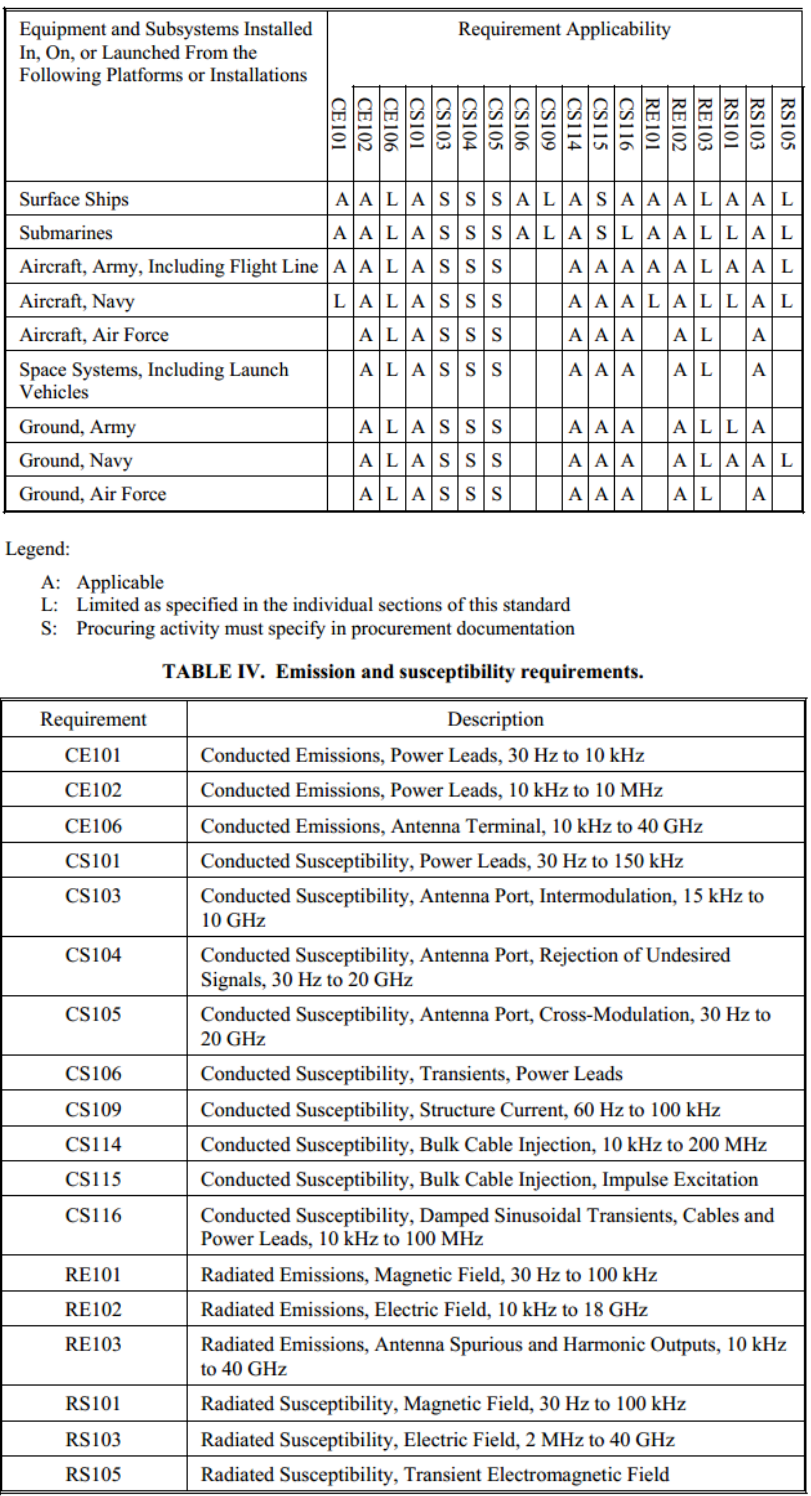

Fig. 2: The requirement matrix, emissions and susceptibility for military.

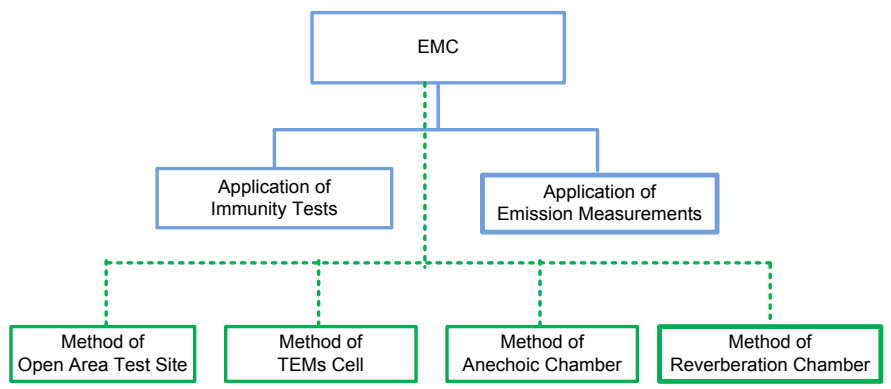

Fig. 3: The two applications and four methods of EMC.

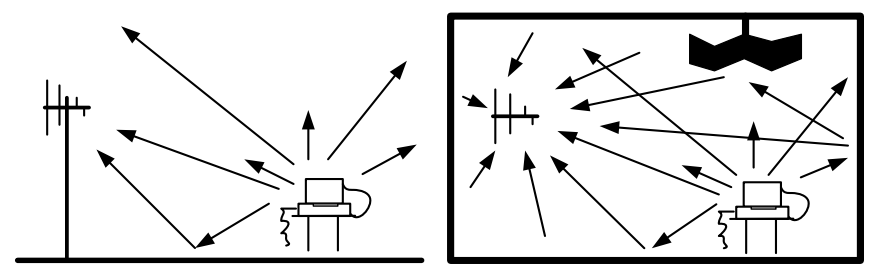

a) Open Area Test Site (OATS) b) Reverberation Chamber (RC) Fig. 4: Example of making emission measurements 


\section{OBJECTIVES}

The quality factor (Q-factor) of a resonant cavity, and thus of the MIMO RC, depends on the losses due to currents in the walls, and due to absorption from antennas. The objective of this research is to study possibility of controlling the composite $Q$-factor below the typical LUF of a RC.

Besides, this research also studies on possibility of scaling the concept model of MIMO RC for the Royal Thai military; i.e., studying on how composite $Q$-factor can affect the sensitivity of MIMO RC when the concept model is scaled up (rescaling to HF range for the military). The outcome of this research could be for making a MIMO RC for the military.

\section{METHODOLOGY AND EXPERIMENTS}

To investigate behavior of composite $Q$-factor, a shielded room which is 5 times bigger than the prototype of MIMO RC (Fig. 6) is modeled on HFSS simulation program. This is just as same as scaling of a dipole antenna; i.e., if a dipole is scaled up for $\mathrm{X}$ times, its operation frequencies will be scaled down for exactly $\mathrm{X}$ times. The shielded room is 5 times bigger than the prototype model of MIMO RC; they are modeled with ANSOFT HFSS simulation program as shown Fig. 7. Characteristics of both models are summarised in Table I.

According to [9], the composite Q-factor $\left(Q_{g}\right)$ can be defined as (3).

$$
Q_{g}=\frac{f_{m n p}}{\Delta f_{m n p}}
$$

where: $f_{m n p}=$ modal frequency, $\Delta f_{m n p}=$ the modal bandwidth, which is determined by the $3 \mathrm{~dB}$ fall of the $\mathrm{S}_{21}$ parameter.

\section{A. Composite Q-factor by Calculation}

The comparisons in Table I show simulated $Q_{g}$ of both enclosures are not scaled for each other. According to [9], $Q_{g}$ is combination of the effect of the walls $\left(Q_{1}\right)$ and effect to of the receiving antenna $\left(Q_{2}\right)$. The $Q_{g}$ can be calculated as:

$$
\begin{aligned}
Q_{g} & =\frac{Q_{1} Q_{2}}{Q_{1}+Q_{2}} \\
Q_{1} & =\frac{3}{2} \frac{V}{S} \sqrt{\frac{\omega_{0} \mu_{0} \sigma}{2 \mu_{r}}} \\
Q_{2} & =\frac{2}{\pi} \frac{\omega_{0}^{3}}{c^{3}} V
\end{aligned}
$$

where: $\mathrm{V}=$ Volume, $\mathrm{S}=$ Surface of the cavity, $\sigma=$ the electric conductivity, $\mu_{0}=$ the permeability of free space, $\mu_{r}=$ relative magnetic permeability, $\omega_{0}=$ angular frequency.

Fig. 8 shows plots for composite the Q-factor $\left(Q_{g}\right)$ for the scaled model (lower frequency axis, black curve) and the full sized shielded room (upper frequency axis, blue curve) with dimensions 5 times larger. The same modes appear at 5 times the frequency in the scaled model as in the full size shielded room, and thus appear at the same place along the horizontal axis in Fig. 8. At low order modes (low frequencies) the composite Q-factor is dominated by $Q_{2}$ and is not affected by scaling (5 times larger size results in a 125 times larger volume, and this is compensated the influence of $\omega^{3}$ ) At higher frequencies, where the composite Q-factor is dominated by $Q_{l}$, the scaling affects the results, and generally a larger room has a higher Q-factor for the same mode. This can also be explained by the equations (4), (5), and (6).

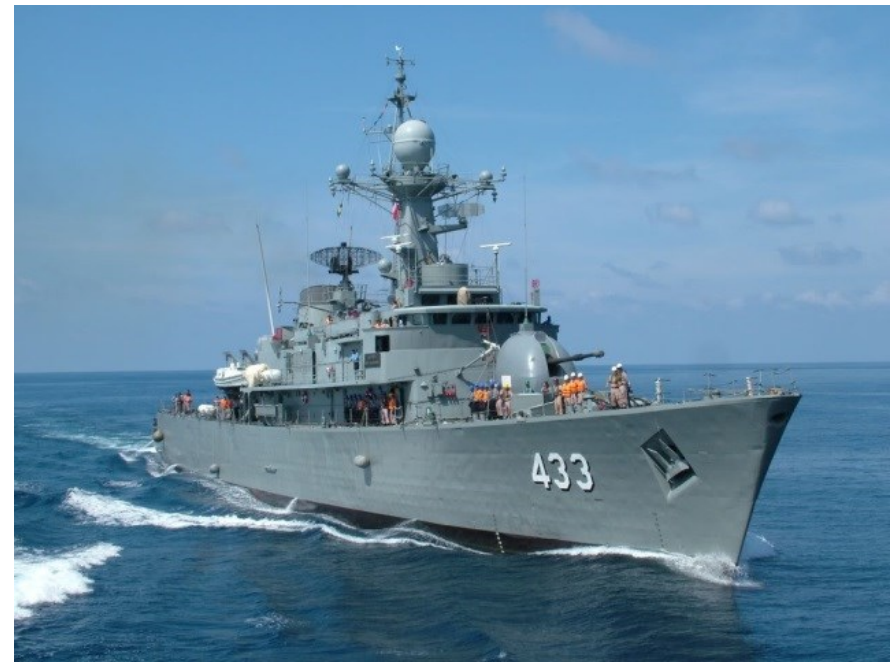

Fig. 5: HTMS. Makutrajakumarn, Royal Thai Navy.

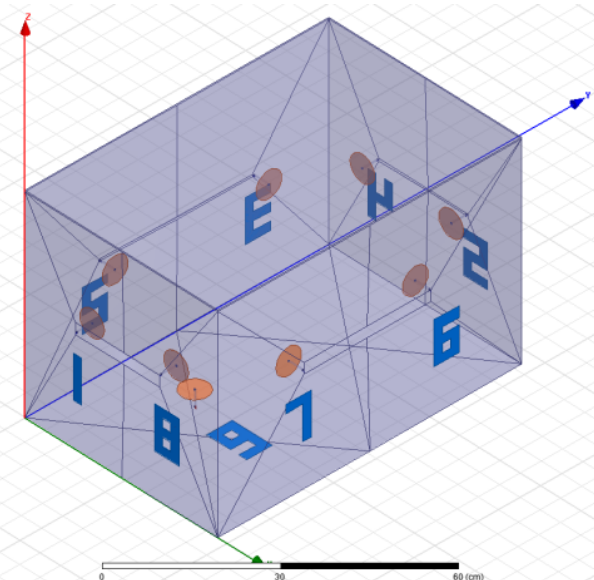

Fig. 6: The model concept of MIMO RC (using of multiple antennas instead of only one)

\section{B. Analysis the results}

The $Q_{1}$ of both enclosures are not in proportional to each other. Instead, at any on X-axis, the $Q_{1}$ of the shielded room is equal to $Q_{1}$ of the model MIMO RC multiplied by $\sqrt{5}$. Indeed, they are not in linear scaled.

$$
\begin{aligned}
Q_{1}=\frac{3}{2} \frac{V}{s} \sqrt{\frac{\omega_{0} \mu_{0} \sigma}{2 \mu_{r}}} & \rightarrow Q_{1}=\frac{V}{s} \sqrt{f_{0}} \rightarrow Q_{1}=\frac{5 \times 5 \times 5}{5 \times 5} \sqrt{\frac{1}{5}} \\
& \rightarrow Q_{1}=\frac{5}{\sqrt{5}}=\sqrt{5}
\end{aligned}
$$

In contrast, the $Q_{2}$, is not affected from neither the electric conductivity $(\sigma)$ nor relative magnetic permeability $(\mu r)$. In fact, the $Q_{2}$ is affected by properties of the antenna (hence the $\omega^{3}$ term) and the total energy in the room (hence the $V$ ); so, their $Q_{2}$-values are linearly scaled (inversely proportional) to each other. For instance, at any on $\mathrm{X}$-axis, the $Q_{2}$ of the scale model will equal to $Q_{2}$ of the shielded room divided by 5 .

Where $Q_{1}$ their $Q_{g}$ which is combination between $Q_{1}$ and $Q_{2}$ cannot be linearly scaled. In other word, if two enclosures are scaled, the bigger room has got higher $Q_{g}$ at every scaling frequency, and the different also depends on type of material.

\section{Controlling the Composite Q-factor on MIMO RC}

The analysis reveals possibility to control $Q_{g}$ by either changing value of $Q_{1}$ (related to walls and thus not practical) 
or value $Q_{2}$ (related to antennas). The model of prototype MIMO RC installed with 9 antennas [1, 2]; therefore, changing value of $Q_{2}$ can be done easier by changing the load on antenna ports, for instance leaving them open, terminating them with $50 \mathrm{ohm}$, matching them for maximum power absorption. E.g. open ports can increase values of $Q_{2}$ compared to $50 \mathrm{ohm}$ loads. Fig. 9 shows results that confirm this reasoning; the composite $Q$-factors $\left(Q_{g}\right)$ are higher when all but one antenna (the one used to measure $S_{12}$ ) are open. unused antennas are opened instead of terminated with 50 ohm loads. Thus, this can mean that more sensitive measurement on MIMO RC can be done when the unused antenna ports are opened.

\section{CONCLUSION}

Method of RC requires high composite $Q$-factors $\left(Q_{g}\right)$ to make more sensitive measurement, but it forces $\mathrm{RC}$ to be usable at only high frequencies. Although novel method, MIMO RC, can be used at lower frequencies than a conventional RC, the sensitivity can by quite low mainly because of power absorbed by antennas.

It is found in this research that $Q_{g}$ on MIMO RC can be increased when the rest of antennas are opened (without any load terminated). This can be explained by (4) that when antennas is unloaded Coefficient relative to the receiving antenna $\left(Q_{2}\right)$ can be increased resulting in increasing of $\left(Q_{g}\right)$. As the results, MIMO RC can make more sensitive measurement. The outcome shows promising possibility of MIMO RC for military concern since $\mathrm{HF}(2 \mathrm{MHz})$ to $\mathrm{Ku}$ band (up to $18 \mathrm{GHz}$ ).

\section{REFERENCES}

[1] C. Choeysakul, F. Schlagenhaufer, and P. Hall, "Characterisation an Design of a Reverberation Chamber for Electromagnetic Emission Measurements for Radio Astronomy Applications," EMC Society of Australia Newsletter, Issue Number 59, Dec. 2012.

[2] C. Choeysakul, F. Schlagenhaufer, and P. Hall, "Reducing the LUF of a Reverberation Chamber based on the concept of MIMO for Electromagnetic Emission Measurements for Radio Astronomy Applications," Asia-Pacific Symposium; APEMC, Melbourne, May 2023, 2013.

[3] The Forrestal Fire, July 29, 1967 Ship's Logs Contributed by Ken Killmeyer, USS Forrestal Association Historian, aboard July 29, 1967, [cited Sep 26, 2013]. Available from URL: http://www.navsource.org/archives/02/cv-59/59f-0729.htm.

[4] T.J. Duggan, "Military Aircraft Electromagnetic Compatibility: Release to Service Testing in the United Kingdom, Past, Present and Future," IEEE International Symposium on Electromagnetic Compatibility 2007: EMC 2007, Honolulu, July 9-13, 2007.

[5] "Requirement for the Control of Electromagnetic Interference Characteristics of Subsystems and Equipment," MIL-STD-461F, Department of Defense Interface Standard, USA, 2007.

[6] "Reverberation chamber test method," IEC 61000-4-21:2011, EMC, Part 4-21: Testing and Measure Technique, April 2011.

[7] A. Walters, T. Doan, and C. Denton, "Field Characterisation of an Aircraft Sized TEM Cell," Electromagnetic Compatibility Symposium, Adelaide, Aug 16-18, 2009, page 47-52.

[8] C. Wilson, "RFI Standards for Equipment to be Deployed on the MRO," February 2009, [Online]. Available: http://snebulos.mit.edu/projects/mwa/file cabinet/0/01080/01080_r05 m.pdf.

[9] P. Besnier, and b. Démoulin, "Electromagnetic Reverberation Chambers," ISTE Ltd, London, 2011, pp. 135-191.
TABLE I

SIMULATED COMPOSITE Q-FACTOR FOR STEEL, AND COPPER

\begin{tabular}{llll}
\hline \hline \multicolumn{1}{c}{ Parameters } & \multicolumn{1}{c}{ Shielded Room } & $\begin{array}{l}\text { Prototype of } \\
\text { MIMO RC }\end{array}$ & Scale \\
\hline Dimensions & $3.6 \mathrm{~m} \times 2.4 \mathrm{~m} \times 2.4 \mathrm{~m}$ & $0.76 \times 0.48 \times 0.48 \mathrm{~m}$ & \\
Surface of cavity & $48.81 \mathrm{~m}^{2}$ & $1.8432 \mathrm{~m}^{2}$ & $1: 5.15$ \\
Volume of cavity & $22.61 \mathrm{~m}^{3}$ & $0.1759 \mathrm{~m}^{3}$ & $1: 5.02$ \\
The $1^{\text {st }}$ resonance & $75 \mathrm{MHz}$ & $375 \mathrm{MHz}$ & $1: 5$ \\
Conventional LUF & $242 \mathrm{MHz}$ & $1,214 \mathrm{MHz}$ & $1: 5$ \\
FOI & DC to $600 \mathrm{MHz}$ & DC $3,000 \mathrm{MHz}$ & $1: 5$ \\
\hline Composite $Q$-Factor & & & \\
STEEL : start FOI & 28.33 & 25.73 & $1.10: 1$ \\
$\left(\mu_{\mathrm{r}}=10\right)$ stop FOI & 903.48 & 712.50 & $1.26: 1$ \\
COPPER: start FOI & 28.56 & 26.17 & $1.09: 1$ \\
\multicolumn{1}{c}{$:$ stop FOI } & 903.48 & 955.86 & $1.09: 1$ \\
\hline \hline
\end{tabular}

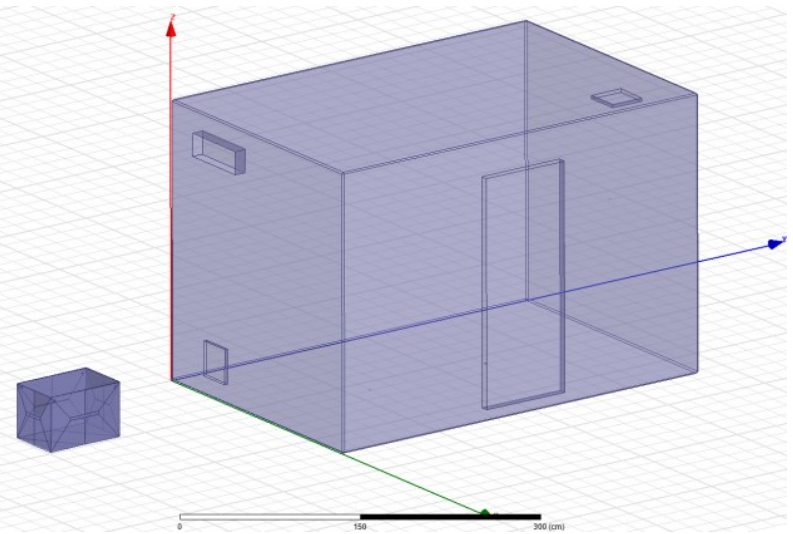

Fig. 7: Models of the prototype of MIMO RC and the shielded room (5 times bigger) on ANSOFT HFSS.

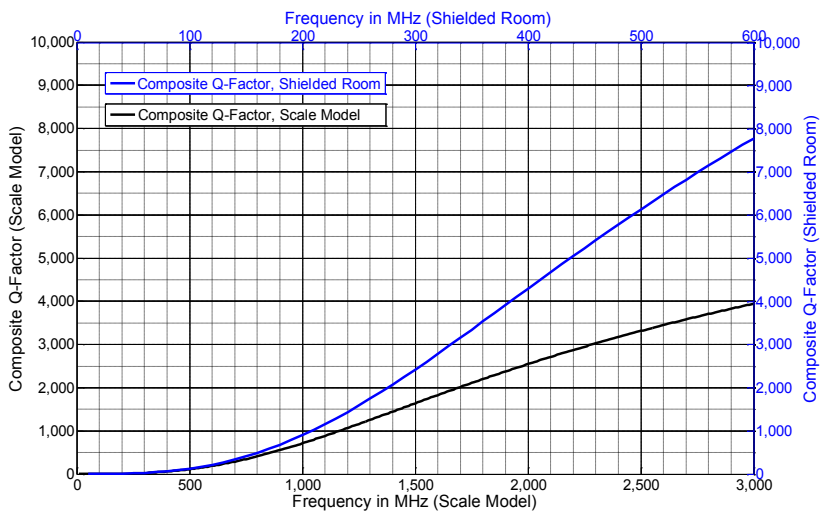

Fig. 8: The double plots show the calculated composite $Q$-factors $\left(\mathrm{Q}_{\mathrm{g}}\right)$ of the prototype MIMO RC and the shielded room.

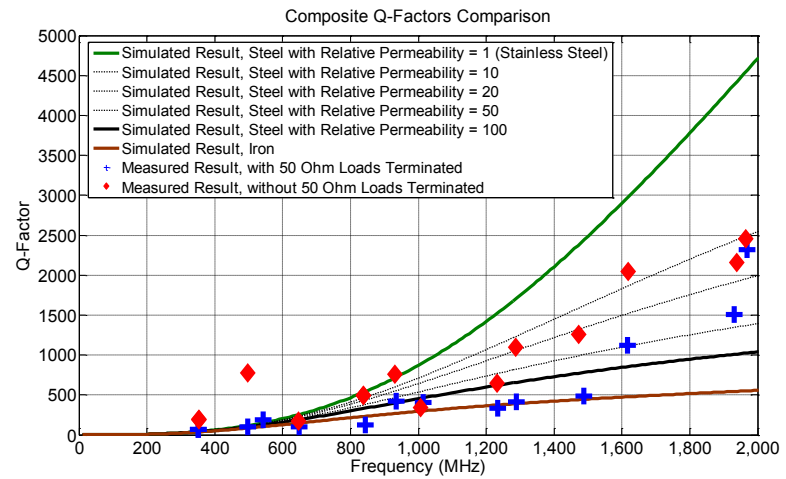

Fig. 9: Comparison between measured and calculated composite QFactors, the 1:5 scale model. 